\title{
Utilizing a Community Dance to Hold STD Testing for Chlamydia and Gonorrhea in High-Risk Adolescents
}

\author{
Andrea Jones, MD | Sara Marlatt, MD | Jenenne Geske, PhD | Birgit Khandalavala, MD
}

PRiMER. 2021;5:30.

Published: 9/2/2021 | DOI: 10.22454/PRiMER.2021.785793

\section{Abstract}

Introduction: Sexually transmitted diseases (STDs) in adolescents are a major public health concern, particularly in underserved communities. While STD screening is recommended by the United States Preventive Services Task Force, limited access remains one of a number of barriers. Community-based approaches may provide greater access and enhance screening rates. This study occured in a nationally recognized hot spot for STDs. We used a unique approach of hosting supervised dances at a community venue, during which free STD screening was offered at an attached health care clinic.

Methods: A series of six Friday night dances was held at one community center sponsored by a nonprofit, girl-focused organization from 2018 to 2019. Dance participants could access an on-site health clinic for self-collected screening for chlamydia and gonorrhea. Treatment was provided for detected STDs. STD screening was also available to area youth at the on-site clinic during regular daytime clinic hours.

Results: A total of 118 adolescents were screened during the school year, and were predominantly female (88.13\%) and African American (85.6\%). More than half of the total STD screenings (51.7\%) were administered during the dances, doubling the total number of STD screenings when compared to those administered during regular clinic hours. A significantly younger cohort were screened at the dances (mean age 14.8 years) compared to those screened at the regular clinic (mean age of 18.9 years). Conclusion: A youth-centric event utilizing community resources can be used to facilitate and enhance screening rates for STDs in youth in an underserved, high-risk community.

\section{Introduction}

In the United States, mitigating sexually transmitted diseases (STDs) in adolescents is critically important. ${ }^{1}$ Almost $40 \%$ of high school students have initiated sexual intercourse, and the earlier the age of sexual onset, the higher the risk of contracting an STD. ${ }^{2,3}$ Left untreated, STDs are associated with serious adverse health outcomes, such as pelvic inflammatory disease, infertility, and cancer. ${ }^{4,5}$ The United States Preventive Services Task Force recommends screening all sexually active women 24 years old and younger for STDs. ${ }^{4}$

In younger adolescents, asymptomatic spread of STDs is common, particularly in underserved areas, leading to an even higher incidence of STDs. ${ }^{6}$ Embarrassment and invasive testing procedures may lead to screening reluctance and a higher STD incidence. ${ }^{7}$ Hence, providing confidential and self-directed STD screening in 
adolescents in underserved areas may be critical. Using community venues is one way to improve access to STD testing. ${ }^{8}$

We undertook a pilot study to determine if STD screening for chlamydia and gonorrhea of high-risk adolescents could be improved by extending access to testing during a community event. The study area is a nationally documented hot spot for STDs, with one of the highest incidences in the United States. ${ }^{7}$ In the county where the study took place, health data from 2018 reports a chlamydia rate of 669.5 per 100,000 residents compared to 539.9 nationally, and a gonorrhea rate of 294.4 compared to 179.1 nationally. ${ }^{9}$ We report an innovative approach to testing that was structured around youth dances held at a community venue.

\section{Methods}

This cross-sectional study used deidentified data. The University of Nebraska Institutional Review Board determined that the study did not constitute human subject research as defined by 45CFR46.102, and was exempt from review.

Six Friday night (8 pm to $12 \mathrm{am}$ ) dances took place in 2018 and 2019 at a nonprofit, girl-focused community center, located in an urban underserved area. The center has an attached primary care clinic, directed by a family physician, that provides free, self-directed STD testing and treatment. Locally, there is limited sex education taught in the public school system. Therefore, the community center uses outreach programs to encourage youth to get tested and provide information to support reproductive well-being.

Dances were open to adolescent girls and boys and were promoted via social media, flyers distributed at high schools and sporting events, and via word of mouth between youth while at school. Community center staff supervised the dances, and attendees could not leave the premises unless signed out by a parent or guardian.

During the first half of the dances, attendees were free to obtain STD testing for chlamydia and gonorrhea from medical personnel at the on-site clinic. Teens had the option of sample self-collection using a genital swab or urine collection. Nucleic acid amplification tests (NAAT) were conducted to detect chlamydia and gonorrhea pathogens. Immediate treatment was provided on-site and free of charge for any positive screen. Clinic staff were available for follow-up visits, questions, consultation, and STD education.

Incentives for STD screening, including candy, lip gloss, and \$5 gift cards were offered. Free condoms were also available.

\section{Results}

During the study period, 118 teens received STD screening at the on-site primary care clinic (Table 1). Sixty-one of these teens $(51.7 \%)$ were tested during the dance hours, and $57(48.3 \%)$ were tested during daytime, regular clinic hours. Most tested were females ( $n=104,88.1 \%)$ and African American $(n=101,85.6 \%)$. Of those tested during the dances, $95.1 \%(n=58)$ were African American, compared to $75.4 \%(n=43)$ of those tested during regular clinic hours.

The average age of all youth screened was 16.7 years $(S D=2.8)$; those who were screened at the dances (mean=14.8, SD=1.4) were significantly younger than those screened during regular clinic hours (mean=18.8, $S D=2.5 ; P<.001)$. The rate of positive STD tests for those screened at the dances $(1.6 \%)$ is significantly smaller than rate of positive tests for those who were screened during regular clinic hours $(14.0 \% ; P=.014)$. 


\section{Conclusions}

Incorporating community events and venues can increase STD screening. ${ }^{8}$ The community center in this study has the unique advantage of having an on-site family medicine clinic, allowing us to evaluate how this collaboration may impact STD screening in adolescents in a high-risk area. Over half of the total STD screenings (51.7\%) were administered during the dances, doubling the total number of STD screenings when compared to only those administered during regular clinic hours. Although one parent became upset to learn that free condoms were available, overall, there was a positive response to our efforts. Teens who obtained the screening during dance hours were significantly younger than those screened during regular clinic hours. Younger teens may have found that the ability to self-swab, a lack of parental supervision, the normalization of screening among peers, and ease of access provided at the dances may have been more appealing. ${ }^{7}$

Screening at a younger age may be critical for STD prevention prior to the onset of multiple STDs. ${ }^{3}$ While our sample yielded low positive rates for STDs, particularly among the younger teens who were screened at the dances, visits to the clinic may have still benefited the attendees as health professionals were able to discuss prevention, provide counseling, and address HPV vaccination with a group that may have otherwise not been exposed to this information. ${ }^{2,3}$

Our study was limited by the small number of males who were screened, especially given that males are more likely to spread STDs. ${ }^{10}$ The dances seemed to be more attractive to teenage girls than boys, perhaps because the community center is targeted toward girls. Recruitment in studies of younger teens tend to skew towards females. ${ }^{11}$ Different recruitment strategies and a wide variety of events may help increase recruitment rates for males, expand participant age range, and ensure increased racial and ethnic diversity. ${ }^{12}$

Additional limitations included the fact that the clinic was not open during the entire dance, limiting screening opportunities. Also, the number of dance attendees is unknown and we are unable to determine the screening rate of dance attendees.

Our study provides preliminary evidence that a youth-centric approach that provides nontraditional access for STD testing at a community center with an on-site health clinic can be useful. We encourage the continued development of effective recruitment and entertainment approaches at community venues to improve the sexual health of youth.

\section{Tables and Figures}


Table 1: Results of Sexually Transmitted Disease Screening

\begin{tabular}{|l|c|c|c|c|}
\hline \multicolumn{1}{|c|}{ Participants } & $\begin{array}{c}\text { Total } \\
\text { Screenings }\end{array}$ & $\begin{array}{c}\text { During the } \\
\text { Dance }\end{array}$ & $\begin{array}{c}\text { During Regular } \\
\text { Clinic Hours }\end{array}$ & $\begin{array}{c}\text { Significance } \\
\text { P Value }\end{array}$ \\
\hline $\mathrm{N}(\%)$ & $118(100)$ & $61(51.7)$ & $57(48.3)$ & .136 \\
\hline Sex $\mathrm{n}(\%)$ & & & & .398 \\
\hline Female & $104(86.4)$ & $52(85.2)$ & $52(91.22)$ & \\
\hline Male & $14(16.5)$ & $9(14.8)$ & $5(8.8)$ & \\
\hline Race, $\mathrm{n}(\%)$ & & & & .004 \\
\hline African American & $101(85.6)$ & $58(95.1)$ & $43(75.4)$ & \\
\hline Caucasian/White & $13(11.0)$ & $3(4.9)$ & $10(17.5)$ & \\
\hline Other & $4(7.0)$ & $0(0)$ & $4(7.0)$ & $<.001$ \\
\hline Mean age in years (SD) & $16.7(\mathrm{SD}=2.8)$ & $14.8(\mathrm{SD}=1.4)$ & $18.8(\mathrm{SD}=2.5)$ & .014 \\
\hline Positive screenings, $\mathrm{n}(\%)$ & $9(7.6)$ & $1(1.6)$ & $8(14.0)$ & \\
\hline
\end{tabular}

\section{Acknowledgments}

The authors acknowledge Frauke Wilcox for administrative assistance, and the Girls, Inc. staff and medical provider working the events. They also acknowledge the Women's Fund of Omaha and Adolescent Health Project for funding.

\section{Corresponding Author}

Birgit Khandalavala, MD

University of Nebraska Medical Center, Department of Family Medicine, 983075 Nebraska Medical Center, Omaha, NE 68198-3075. 402-559-3864. Fax: 402-559-6051

birgit.khandalavala@unmc.edu

\section{Author Affiliations}

Andrea Jones, MD - Department of Family Medicine, University of Nebraska Medical Center, Omaha, NE Sara Marlatt, MD - Department of Family Medicine, University of Nebraska Medical Center, Omaha, NE Jenenne Geske, PhD - Department of Family Medicine, University of Nebraska Medical Center, Omaha, NE Birgit Khandalavala, MD - Department of Family Medicine, University of Nebraska Medical Center, Omaha, NE

\section{References}

1. Sexually Transmitted Diseases (STDs). Centers for Disease Control and Prevention. Updated 2021. Accessed February 10, 2021.https://www.cdc.gov/std/default.htm.

2. Tu W, Batteiger BE, Wiehe S, et al. Time from first intercourse to first sexually transmitted infection diagnosis among adolescent women. Arch Pediatr Adolesc Med. 2009;163(12):1106-1111. doi:10.1001/archpediatrics.2009.203

3. Kann L, McManus T, Harris WA, et al. Youth risk behavior surveillance-United States, 2017. MMWR Surveill Summ. 2018;67(8):1-114. doi:10.15585/mmwr.ss6708a1

4. Sexually Transmitted Infections: Behavioral Counseling. United States Preventive Services Taskforce. Published August 18, 2020. Accessed January, 20, 2021. https://www.uspreventiveservicestaskforce.org /uspstf/recommendation/sexually-transmitted-infections-behavioral-counseling

5. Health Professional Shortage Area. Health Resources \& Services Administration. Accessed January 21, 
2021. https://data.hrsa.gov/tools/shortage-area/hpsa-find

6. Tilson EC, Sanchez V, Ford CL, et al. Barriers to asymptomatic screening and other STD services for adolescents and young adults: focus group discussions. BMC Public Health. 2004;4(1):21. doi:10.1186/1471-2458-4-21

7. Islam KM, Vinson L, Nared S, et al. Combating STI epidemic: community perspectives learned from mixed-gender focus group study. Int Sch Res Notices. 2013.

8. Wright S, Valentine JA, Hayes NA, Johnson DB, Arauz NR. Community based approaches to reducing STDs: Community Engagement Toolkit. Atlanta: Centers for Disease Control and Prevention; 2019. Accessed January 25, 2021. https://www.cdc.gov/std/health-disparities/cars-toolkit-2020.pdf

9. STDs in Douglas County. Douglas County Health Department. Updated 2020. Accessed August 6, 2021. https://www.dchealthdata.com/images/top_area/STD2019_short_for_Web.pdf

10. Workowski KA. Centers for disease control and prevention sexually transmitted diseases treatment guidelines. Clin Infect Dis. 2015;61(suppl 8):S759-S762. doi:10.1093/cid/civ771

11. Ott MA, Campbell J, Imburgia TM, Yang Z, Tu W, Auerswald CL. Community engagement and venuebased sampling in adolescent male sexually transmitted infection prevention research. J Adolesc Health. 2018;62(3S):S58-S64. doi:10.1016/j.jadohealth.2017.10.002

12. Bradley ELP, Lanier Y, Ukuku Miller AM, Brawner BM, Sutton MY. Successfully recruiting black and Hispanic/Latino adolescents for sexually transmitted infection and HIV prevention research. J Racial Ethn Health Disparities. 2020;7(1):36-44. doi:10.1007/s40615-019-00631-7

Copyright $@ 2021$ by the Society of Teachers of Family Medicine 